\title{
Linearity improvement of differential CMOS low noise amplifier
}

\author{
Maizan Muhamad ${ }^{1}$, Norhayati Soin ${ }^{2}$, Harikrishnan Ramiah ${ }^{3}$ \\ ${ }^{1}$ Faculty of Electrical Engineering, Universiti Teknologi MARA, ShahAlam, Malaysia \\ ${ }^{1,2,3}$ Faculty of Engineering, University of Malaya, Kuala Lumpur, Malaysia
}

\section{Article Info \\ Article history: \\ Received Sep 30, 2018 \\ Revised Nov 27, 2018 \\ Accepted Dec 15, 2018}

\section{Keywords:}

CMOS

Differential

Linearity

Low noise amplifier (LNA)

Noise figure (NF)

\section{Corresponding Author:}

Maizan Muhamad,

Faculty of Electrical Engineering,

Universiti Teknologi MARA,

40450, ShahAlam, Malaysia.

Email: maizan@salam.uitm.edu.my

\begin{abstract}
This paper presents the linearity improvement of differential CMOS low noise amplifier integrated circuit using 0.13um CMOS technology. In this study, inductively degenerated common source topology is adopted for wireless LAN application. The linearity of the single-ended LNA was improved by using differential structures with optimum biasing technique. This technique achieved better LNA and linearity performance compare with single-ended structure. Simulation was made by using the cadence spectre RF tool. Consuming $5.8 \mathrm{~mA}$ current at $1.2 \mathrm{~V}$ supply voltage, the designed LNA exhibits S21 gain of $18.56 \mathrm{~dB}$, noise figure (NF) of $1.85 \mathrm{~dB}, \mathrm{~S} 11$ of $-27.63 \mathrm{~dB}, \mathrm{~S} 22$ of $-34.33 \mathrm{~dB}, \mathrm{~S} 12$ of $-37.09 \mathrm{~dB}$ and IIP3 of $-7.79 \mathrm{dBm}$.
\end{abstract}

Copyright $\odot 2019$ Institute of Advanced Engineering and Science. All rights reserved.

\section{INTRODUCTION}

Nowadays, complementary metal oxide semiconductor (CMOS) widely used in designing wireless system because of the low cost and easy integration. Low noise amplifier (LNA) is the first building block in the wireless system. Its main purpose is to provide gain while with minimum noise as possible to the network. As the first active block in the receiver chain, the performance of an LNA controls the overall performance of receivers. Noise performance and power gain are the most important characteristics of an LNA. Beside these characteristics, the main parameters affecting the selection of a proper circuit for an LNA are DC power consumption, bandwidth, stability, linearity, supply voltage and chip area.

Even though single-end LNA input stage consumes less power and the active chip area consumption is minimum, differential topology is preferred for the following important issues [1]. Since the noise figure is the critical factor for the LNA, by using differential signalling can offer better noise performance due to the ability of rejecting the common-mode noise. Theoretically, the two sides of the circuit are identically matched and therefore the common-mode noise of each side can be viewed as the same. Secondly, not only limiting towards the noise but also for the linearity performance, the differential mode amplifier exhibits better performance [2]. Due to the inherent circuit architecture which is symmetrical, the nature ability of cancelling the even-order distortions increases the linearity of the amplifier.

The signal to noise will be degraded due to the insufficient linearity of a LNA. Therefore, having a high linearity, IIP3 LNA can reduce the degradation of carrier to noise [3]. This linearity should not be at the expenses of gain or noise figure, NF.

In integrated analogue electronics and especially in RF applications, a fully differential approach is usually preferred, due to its well-known properties of immunity to common-mode disturbances, rejection to 
parasitic couplings and increased dynamic range [3]. Although the differential operation must be preserved in the chip, there are cases where the input signal is single-ended such as RF image filters and IF filters in a RF receiver. In addition, there are circuits that require differential signals to perform their function. In these situations, a stage which can convert single-ended to differential signals is needed. Several highly integrated direct-conversion CMOS front-ends achieve high performance, but at the cost of requiring a fully differential LNA that needs two RF input pins and an external RF single-ended to differential conversion [4]. This typically requires special front-end filters or an additional off-chip balun, which can incur extra loss and can degrade the system noise figure $[5,6]$.

\section{RESEARCH METHOD}

The LNA is to be designed with the following proposed specification summarized in Table 1. Generally, the $0.13 \mu \mathrm{m}$ CMOS LNA is to be designed such as to comply with the wireless local area network (WLAN) specifications whereby the LNA needs to provide a high gain with low noise figure at low power, with its centre operating frequency at $2.4 \mathrm{GHz}$.

Table 1. LNA Design Specification for WLAN Standard

\begin{tabular}{cc}
\hline Parameter & Target specification \\
\hline CMOS Process technology & $0.13 \mu \mathrm{m}$ \\
Frequency band & $2.4 \mathrm{GHz}$ \\
Power supply & $\leq 1.2 \mathrm{~V}$ \\
Gain, $\mathrm{S}_{21}$ & $>15 \mathrm{~dB}$ \\
Input matching $\left(\mathrm{S}_{11}\right)$ & $<-12 \mathrm{~dB}$ \\
\hline
\end{tabular}

This LNA is constructed using an inductively degenerated cascode topology. The cascode topology is adopted as it provides high gain and good input-output isolation, which improve circuit design stability and also simplify input matching. The simplified schematic of the proposed CMOS LNA for noise optimization and gain enhancement is illustrated in Figure 1(a) and the simplified small-signal equivalent circuit is shown in Figure 1(b) where $V_{R F}$ and $R_{S}$ model the antenna.

Inductively degenerated topology has the advantage of better control over the value of the real part of the input impedance. The function of the $\mathrm{L}_{\mathrm{s}}$ is to generate the real impedance to match the input impedance to $50 \Omega$ and hence good noise performance [7]. The inductively-degenerated CS LNA is to help the input matching. The small-signal model in Figure 1 (b) is used as a guide in order to determine the input impedance of the circuit. The subsequent derivations can illustrate how the input can be simply matched to the source resistance. $Z_{\text {in }}$ is a RLC series network circuit with a resistive term which is straightly proportional to the value of the inductance. Where at resonance, the real term in $\mathrm{Z}_{\text {in }}$ contains $\mathrm{L}_{\mathrm{s}}$ [8]

$$
\begin{aligned}
& \text { Zin }=\frac{V g}{I g}=\frac{I g R g+V c+j \omega I s L s}{I g} \\
& \text { Zin }=\frac{\text { Ls.gm }}{\text { Cgs }}
\end{aligned}
$$

Where $\mathrm{Z}_{\text {in }}$ is 50 Ohms. In most LNA design the value of $\mathrm{L}_{\mathrm{s}}$ was assumed and the values of $\mathrm{g}_{\mathrm{m}}$ and $\mathrm{C}_{\mathrm{gs}}$ are calculated based on the formula to find the required for $Z_{i n}$. Consequently, in this input matching the utilization of degenerated inductor is needed. This make the presence of $\mathrm{L}_{\mathrm{s}}$ helps in providing a right input impedance to terminate the off-chip RF filter in the preceeding the of the LNA, in which in the typical condition,need to match to $50 \Omega$ impedance. While $\mathrm{L}_{\mathrm{g}}$ will resonate with $\mathrm{C}_{\mathrm{gs}}$ and it guarantees that the input frequency adjusted to the operating frequency of the application. Hence during resonance, the source resistance and others parameter can be determined as the expression derived below [8]:

$$
\omega_{T}=\frac{\mathrm{gm}}{\mathrm{Cgs}}=\frac{\mathrm{Rs}}{\mathrm{Ls}}
$$

Where $\omega_{T}$ is defined as a cut off frequency. The value of the $R_{s}$ is 50 ohms

$$
Q_{L}=\sqrt{1+(1 / \mathrm{p})}
$$


Where $\mathrm{p}=\frac{\sigma \cdot \mathrm{a}^{2}}{5 \cdot \gamma}$

The parameters for the $\mathrm{p}$ usually depend on the RFCMOS Technology, but typically $\gamma$ is set between $2-3, \sigma$ is set to 2-3 times the value of $\mathrm{Y}$ and the $\alpha$ is assumed to be $0.8-1[9]$.

$$
\mathrm{Lg}=\frac{\mathrm{QL} \cdot \mathrm{Rs}}{\omega \mathrm{o}} . \mathrm{LS}
$$

Where $\omega_{0}$ is the center frequency.
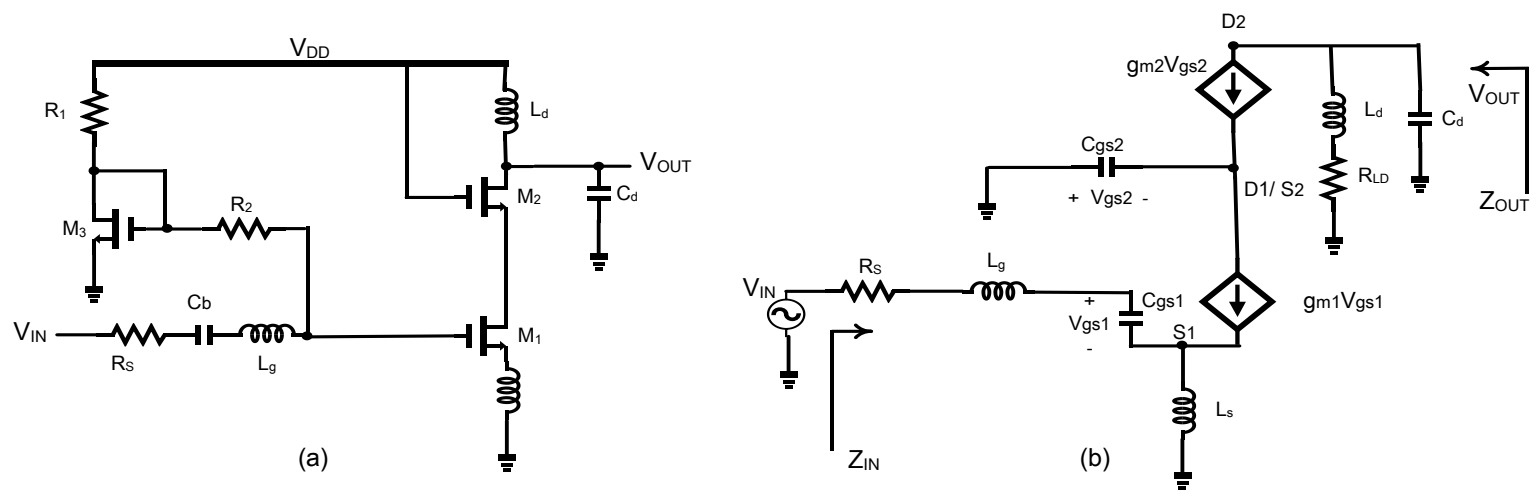

Figure 1. Simplified configuration of (a) the proposed single ended LNA and (b) the small-signal equivalent circuit

Figure 2 illustrates the modified differential LNA. The combination two circuit single ended will produce the double ended. For the sake of simplicity, the biasing circuit of differential LNA is not shown. The gate-source capacitance can be determined by below expression [8]:

$$
\begin{aligned}
& \text { Cgs }=\frac{1}{\omega 0^{2}(L g+L s)} \\
& \text { Cgs }=\frac{2}{3} \text { Cox. W. Lmin }
\end{aligned}
$$

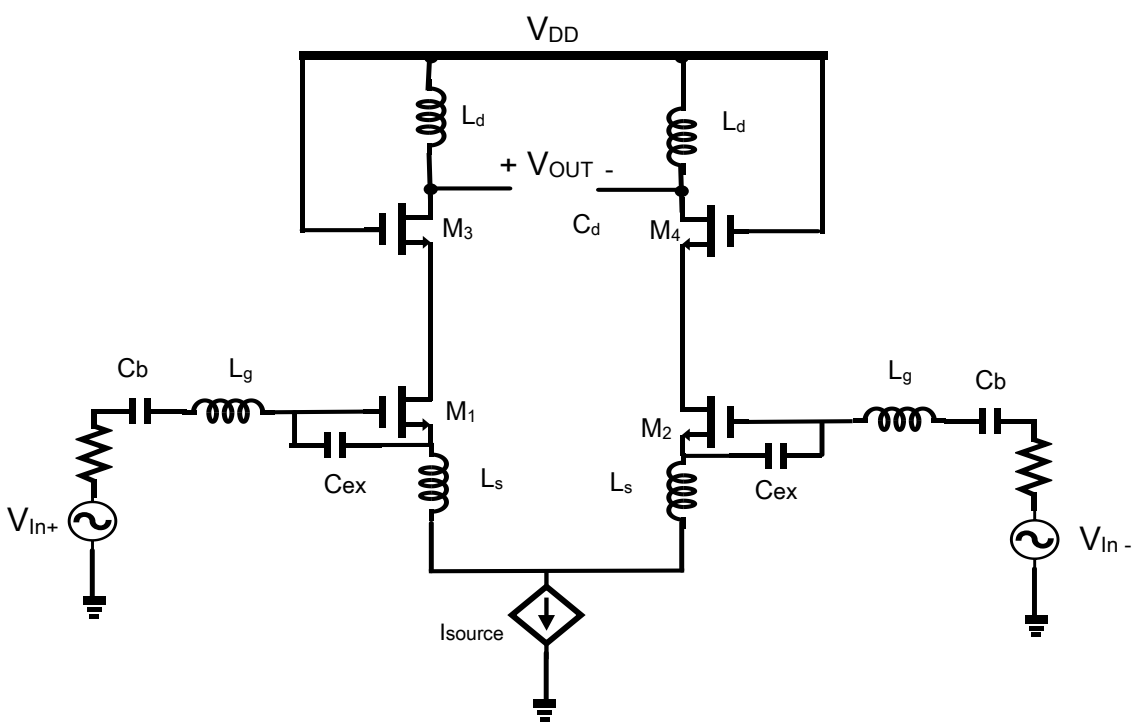

Figure 2. Inductively degenerated common source differential LNA 
Which the width of transistor can be expressed as:

$$
\begin{aligned}
& \mathrm{W}=\frac{3}{2} \frac{\mathrm{Cgs}}{2 \text { Cox.Lmin }} \\
& \varepsilon O \mathrm{X}=\text { EOX. } \varepsilon O
\end{aligned}
$$

Where

$$
\begin{aligned}
& \varepsilon o=\text { dielectric constant forfree space }=\frac{8.854 \mathrm{E}^{-14} \mathrm{~F}}{\mathrm{~cm}} \\
& \varepsilon \mathrm{ox}=\text { dielectric constant for silicon }=3.9
\end{aligned}
$$

\section{RESULTS AND ANALYSIS}

Figure 3 illustrates the simulation of s-parameters and noise figure for both single-ended and differential LNA. The S-parameter plots and noise figure performances are shown in Figure 3. As can be seen from Figure 3, the circuit's input and output were matched to the $50 \Omega$ required at the operating frequency of $2.4 \mathrm{GHz}$. Base from the curve, both structures exhibit good performance and satisfy the design requirement. The input return loss, $\mathrm{S}_{11}$ is $-24.14 \mathrm{~dB}$ and $-27.63 \mathrm{~dB}$ for single-ended and differential respectively. While the output return loss, $S_{22}$ is $-23.47 \mathrm{~dB}$ for single ended and $-34.33 \mathrm{~dB}$ for differential structure. The noise figure for single ended LNA is $1.88 \mathrm{~dB}$ which is not much different with differential structures value $1.85 \mathrm{~dB}$. The attained value of NF is believed to be good as it exceeds the requirement which is typically below $2 \mathrm{~dB}$ without having to trade off the power gain which also satisfies the requirement. Differential LNA obtain greater gain, $\mathrm{S}_{21}$ which is $18.56 \mathrm{~dB}$ as compared to single-ended $17.9 \mathrm{~dB}$.

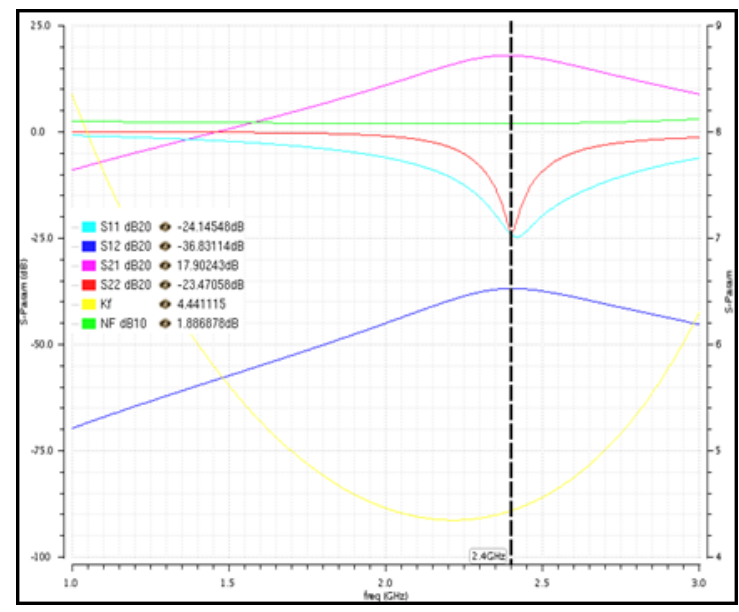

(a)

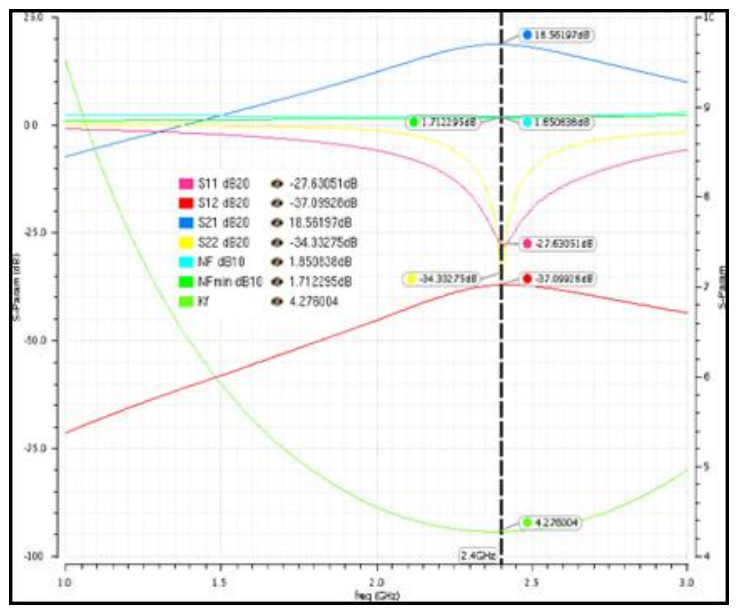

(b)

Figure 3. S-parameters and noise figure performance: (a) single ended LNA, (b) differential LNA

Figure 4 presents the simulation performance of third order intercept point, IIP3. In order to simulate the IIP3, different value of input signal need to supply to the circuit and the fundamental frequency and the third order inter-modulation component. The single ended LNA achieved - $10.6 \mathrm{dBm}$ while the differential manage to get $-7.75 \mathrm{dBm}$. The linearity of the designed LNA improved differential improved by $2.85 \mathrm{dBm}$. 


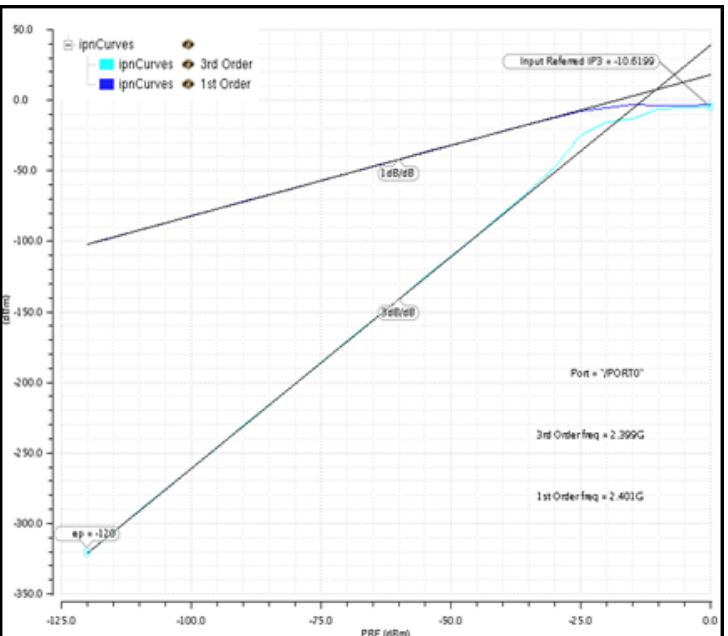

Figure 4. Simulation of Third Order Intercept Point (IIP3): (a) single ended LNA, (b) Differential LNA

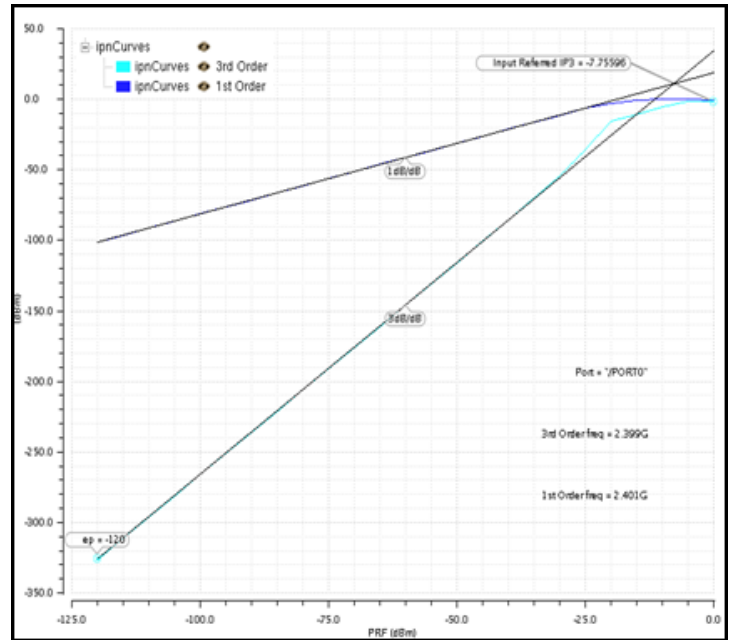

Figure 5 shows the physical representation (layout) of differential LNA using 6 octagonal spiral inductors

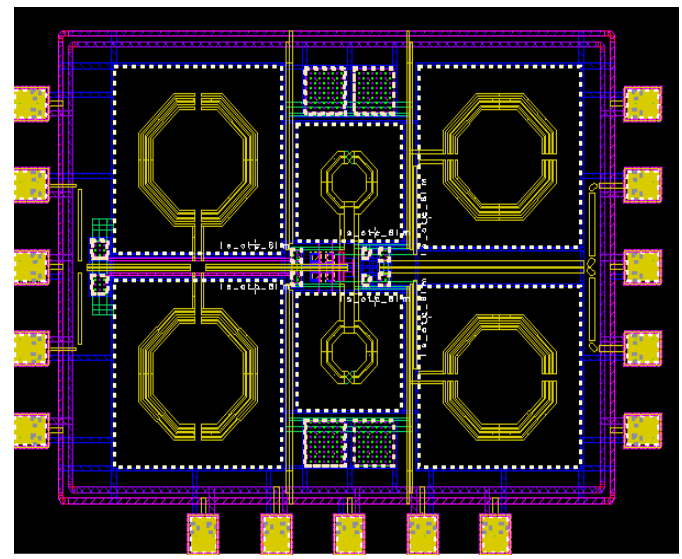

Figure 6. Layout of differential LNA

Table 2 summarize the simulation performance comparison of both structures. By using differential structures, the LNA achieved better linearity without trade off its noise figure and gain performance.

Table 2. Performance comparison of with other published work

\begin{tabular}{lcccccc}
\hline LNA structure & $\mathrm{S}_{11}(\mathrm{~dB})$ & $\mathrm{S}_{21}(\mathrm{~dB})$ & $\mathrm{S}_{12}(\mathrm{~dB})$ & $\mathrm{S}_{22}(\mathrm{~dB})$ & $\mathrm{NF}(\mathrm{dB})$ & $\mathrm{IIP} 3(\mathrm{dBm})$ \\
\hline Single-ended & -24.14 & 17.9 & -36.83 & -23.47 & 1.88 & -10.61 \\
Differential & -27.63 & 18.56 & -37.09 & -34.33 & 1.85 & -7.79 \\
\hline
\end{tabular}

\section{CONCLUSION}

In this paper, the design of differential LNA are successfully implemented using Silterra 130-nm CMOS technology. The LNA design exhibits high forward gain $\left(\mathrm{S}_{21}\right)$ of $18.56 \mathrm{~dB}$, high reverse isolation $\left(\mathrm{S}_{12}\right)$ of -37.09 $\mathrm{dB}$ and a good linearity IIP3 of $-7.79 \mathrm{dBm}$ at $2.4 \mathrm{GHz}$. The LNA shows a low NF of $1.85 \mathrm{~dB}$. Linearity of the LNA is improved by using differential structures with optimum biasing technique. The LNA operates at $1.2 \mathrm{~V}$ supply and consumes only $7 \mathrm{~mW}$ of power. 


\section{REFERENCES}

[1] Zokaei A, Amirabadi A. A $65 \mathrm{~nm}$ linear broad-band differential Low Noise Amplifier using post distortion technique. Microelectronics J. 2018;74(August 2017):24-33.

[2] Das DM, Srivastava A, Ananthapadmanabhan J, Ahmad M, Baghini MS. A novel low-noise fully differential CMOS instrumentation amplifier with 1.88 noise efficiency factor for biomedical and sensor applications. Microelectronics J. 2016;53:35-44.

[3] Zhang H, Member S, Sánchez-sinencio E, Fellow L, Paper I. Linearization Techniques for CMOS Low Noise Amplifiers : A Tutorial. 2011;58(1):22-36.

[4] Feng Y, Takemura G, Kawaguchi S, Kinget P. Design of a high performance 2-GHz direct-conversion front-end with a single-ended RF input in $0.13 \mu \mathrm{m}$ CMOS. IEEE J Solid-State Circuits. 2009;44(5):1380-90.

[5] Robens M, Wunderlich R, Heinen S. Differential Noise Figure De-Embedding: A Comparison of Available Approaches. IEEE Trans Microw Theory Tech. 2011 May;59(5):1397-407.

[6] Shim J, Jeong J. A band-selective low-noise amplifier using an improved tunable active inductor for 3-5 GHz UWB receivers. Microelectronics J. 2017;65(May):78-83.

[7] Fan XH, Zhang H, Member S, Sánchez-sinencio E. A noise reduction and linearity improvement technique for a differential cascode LNA. IEEE J Solid-State Circuits. 2008;43(3):588-99.

[8] Microelectronic C, Excellence D, Campus E, Noh NM, Zainal T, Zulkifli A, et al. Systematic Width Determination for the Design of Power-Constrained Noise Optimization Inductively Degenerated Low Noise Amplifier. IETE J Res. 2010;56(5):249-56.

[9] Microelectronic C, Excellence D, Campus E. Systematic Width Determination for the Design of Power-Constrained Noise Optimization Inductively Degenerated Low Noise Amplifier. 2011;56(5):249-56. 